\title{
Compliance to clinical guidelines for early-stage epithelial ovarian cancer in relation to patient outcome
}

\author{
Edith A. Sijmons ${ }^{\text {a }}$, Marieke A.L. van Lankveld ${ }^{\mathrm{b}}$, Petronella O. Witteveen ${ }^{\mathrm{c}}$, \\ Petra H.M. Peeters ${ }^{\mathrm{b}}$, Veronica C.M. Koot ${ }^{\mathrm{b}, \mathrm{d}}$, Jules Schagen van Leeuwen ${ }^{\mathrm{e}, *}$ \\ ${ }^{a}$ University Medical Center Utrecht, Department of Gynaecology, Box 85500, 3508 GA Utrecht, The Netherlands \\ ${ }^{\mathrm{b}}$ Julius Center for Health Sciences and Primary Care, University Medical Center Utrecht, \\ Box 85500, 3508 GA Utrecht, The Netherlands \\ ${ }^{\mathrm{c}}$ University Medical Center Utrecht, Department of Internal Medicine, Box 85500, 3508 GA Utrecht, The Netherlands \\ ${ }^{\mathrm{d}}$ Comprehensive Cancer Center Middle Netherlands, Box 19079, 3501 DB Utrecht, The Netherlands \\ e Sint Antonius Hospital, Department of Gynaecology, Box 2500, 3430 EM Nieuwegein, The Netherlands
}

Received 3 July 2005; received in revised form 2 February 2006; accepted 28 March 2006

\begin{abstract}
Objectives: To assess compliance to current surgical staging and adjuvant treatment guidelines for patients with early-stage epithelial ovarian carcinoma and its impact on overall survival.

Methods: Patients diagnosed between 1991 and 1997 with early-stage ovarian cancer were recruited from the Regional Cancer Registry of the central region in the Netherlands. Demographic data, tumour characteristics, surgical findings and therapeutic data were abstracted from medical records. Patients were classified into optimal and non-optimal surgical staging. Overall survival was estimated using Kaplan-Meier method. To adjust for age hazard ratios for overall survival were estimated with a Cox Proportional Hazards model.

Results: One hundred and twenty-five patients were included in the study, 41 of them (32.8\%) were optimally staged. Guidelines for adjuvant radio- or chemotherapy were adequately followed in all 62 grade I patients and in 44 out of 59 grade II and III patients (74.6\%). During 734.6 person-years of follow up 31 patients died. Five-year overall survival figures were $97.6 \%$ in the optimally staged group and $68.5 \%$ in the nonoptimally staged group. Patients who were non-optimally staged, had a significant higher risk to die than those who were optimally staged (HR: 7.4; 95\% CI: 1.7-32.2). In patients with a grade II and III tumours, complete surgical staging still had a significant influence on survival (HR: $3.8 ; 95 \%$ CI 1.7-8.3).

In women with grade II or III tumours, adjuvant radio- or chemotherapy administered in accordance to the guidelines did not improve overall survival regardless whether they were optimally staged or not.

Conclusion: Incomplete staging in early-stage ovarian cancer leads to gross mis-classification in grade II and III tumours and to a lesser extent in grade I tumours. This leads to undertreatment in both surgical and adjuvant therapy. Subsequently unnecessary deaths may occur. More effort must be put in identifying obstacles interfering with compliance of guidelines
\end{abstract}

(C) 2006 Published by Elsevier Ireland Ltd.

Keywords: Early-stage ovarian neoplasms; Surgical staging; Guideline adherence; Survival

\section{Introduction}

About $20-25 \%$ of all epithelial ovarian carcinomas are diagnosed at an early stage [1]. Overall the prognosis of these cancers is relatively favourable [2].

\footnotetext{
* Corresponding author.
}

Guidelines emphasize the need for optimal surgical staging to minimize the risk of residual disease and thus underdiagnosis and undertreatment $[2,3]$.

Gynecological Working Groups of the Comprehensive Cancer Centers (CCC) take charge of producing and implementing evidence based guidelines of gynecological cancers in The Netherlands. In this context the CCC of the region Middle Netherlands (CCCMN) initiated the guideline 
for surgical staging and adjuvant treatment of ovarian cancer [1].

This study evaluates current treatment practice and assesses overall survival for patients with early-stage ovarian carcinoma with respect to compliance to the guidelines for surgical staging and adjuvant treatment in ovarian cancer, developed by the CCCMN.

\section{Methods}

\subsection{Study population}

The CCCMN-region has 1.3 million inhabitants and is covered by seven hospitals. The Regional Cancer Registry (RCR) of the CCCMN was established in 1986 and is informed by regular announcements of the pathological archive (a nationwide coverage of all histological diagnosis) of new cancer cases in the region. Co-workers of the CCCMN visit all hospitals to extract additional data from medical records of notified cancer cases. Each record in the RCR corresponds to a single tumour and contains among other variables, a unique identifier. Other variables present in the register include date of diagnosis, tumour type according to the International Classification of Diseases for Oncology, first edition (ICD-O-1) before 1993 and ICD-O-2 thereafter; stage of the disease was filed according to the fourth edition of the TNM-Classification of Malignant Tumours [4].

For the present study, all women who were diagnosed between 1991 and 1997, with ovarian carcinoma stage IA, IB or IC according to the International Federation of Gynaecology and Obstetrics (FIGO) were identified in the RCR of the CCCMN (topography code in ICD-O-1: 183 and in ICD-O-2: C56). Two hundred and four women were identified. Women with a borderline malignancy (ICD-O morphology codes $8442,8451,8462,8472$, and 8473) $(n=49)$, a non-epithelial malignancy (ICD-O morphology codes 8310, 8620, 8621, $8622,8890,8891,8896,9060,9071$, and 9080$)(n=22)$, or a simultaneous other primary tumour $(n=6)$ were excluded. Medical records were missing for two women. Finally, 125 women were included in the present cohort. Information on tumour characteristics, clinical stage, therapy and death was collected from medical records. If available, reasons for deviating from the surgical guidelines were also extracted.

\subsection{Guidelines}

The guidelines for epithelial ovarian carcinoma FIGO stage I include both surgical and adjuvant therapeutic procedures.

In case carcinoma is clinically limited to the ovaries, the guideline prescribes extensive surgery to obtain optimal staging. This means in cases where a diagnostic laparoscopy or laparotomy is not conclusive, a second operation is indicated to complete the staging procedure [5].

Optimal staging surgery includes abdominal washings, bilateral salphingo-oophorectomy (BSO), a transabdominal hysterectomy (TAH), infracolic omentectomy, random peritoneal and diaphragm biopsies, with (uni- or bilateral) pelvic and para-aortic lymph node dissection (PLND and PALND).

Young women, who have unilateral cancer and who wanted to preserve fertility, the unattached ovary and uterus can be left in situ. In case of carcinoma in both ovaries, bilateral PLND and PALND is indicated [6].

During the study period in case of a uni-lateral ovarian tumour, a uni-lateral pelvic and para-aortic lymph node dissection was prescribed in our guideline $[7,8]$.

The guideline for adjuvant therapy is based on the histopathological outcome of the surgical specimen. If the tumour is well differentiated (grade I) no adjuvant therapy is indicated. In case of a grade II or III tumour or of a clear cell tumour either adjuvant radiotherapy or chemotherapy is indicated. Our evaluation of treatment is based on the guidelines that were valid in the period that the patients were diagnosed and treated.

At the beginning of the study-period standard adjuvant therapy consisted of radiotherapy if no contraindications were present [9-11]. Chemotherapy was reserved for recurrences. During the study period there was a shift in the guideline towards adjuvant chemotherapy as standard therapy. This switch was made not because of better efficacy, but because of the side effects of radiotherapy.

\subsection{Data collection and analysis}

Surgical staging was classified 'optimal' if the procedure was performed in accordance with the guideline as described earlier; else patients were classified as sub-optimally staged. Patients who had their uterus and one adnex preserved for fertility reasons, but who were fully staged according to the guideline, were classified as optimal.

In patients with grade II and III tumours it was assessed whether or not they had received adjuvant chemo- or radiotherapy as prescribed by the guidelines.

Patients were followed from date of diagnosis until 31st December 2001. Combining information from the RCR, medical records, General Practitioners as well as from municipal registries completed follow-up of vital status. Overall survival estimates were computed by KaplanMeier method. Hazard ratios for overall survival were estimated with a Cox Proportional Hazard model. Ninetyfive percent confidence intervals (CI) were computed. Statistical Package for the Social Sciences, version 11.0 (SPSS Inc., Chicago, IL 60606-6412) was used for statistical analysis.

\section{Results}

Table 1 shows characteristics of the women in the cohort. Mean age is 54.7 years (range 18-89 years) and mean follow-up is 6.1 years (range 1 week to 10.2 years) (Table 2). 
Table 1

Characteristics of 125 women with early-stage ovarian carcinoma, who were identified in de Regional Cancer Registry

\begin{tabular}{lc}
\hline & $n(\%)$ \\
\hline FIGO-stage & \\
Stage 1A & $82(65.6)$ \\
Stage 1B & $2(1.6)$ \\
Stage 1C & $32(25.6)$ \\
Stage 1, NOS & $9(7.2)$ \\
Histological diagnosis & \\
Adenocarcinoma, serous & $37(29.6)$ \\
Adenocarcinoma, mucinous & $49(39.2)$ \\
Adenocarcinoma, NOS & $17(13.6)$ \\
Endometroid carcinoma & $12(9.6)$ \\
Clear cell carcinoma & $5(4.0)$ \\
Other & $5(4.0)$ \\
Histological grade & \\
Grade I & $62(49.6)$ \\
Grade II & $45(36.0)$ \\
Grade III & $14(11.2)$ \\
Missing & $4(3.2)$ \\
\hline NOS: not otherwise specified
\end{tabular}

NOS: not otherwise specified.

Optimal staging was performed in $32.8 \%$ of the patients $(n=41)$, whereas in the remaining 84 women different combinations of surgical procedures were performed, resulting in non-optimal staging (Table 3 ).

In 12 patients, the uterus and contra-lateral ovary were preserved for fertility reasons. Over $50 \%$ of women did not obtain PLND and PALND after ovarian cancer had been diagnosed.

In 69 patients $(82 \%)$ no explanation for sub-optimal staging could be extracted from the medical records; for seven patients the gynaecologist considered the first procedure to be adequate, seven patients were not reoperated because of poor physical health and one patient refused a second staging operation.

Fig. 1 shows a flow chart of staging results and adjuvant treatment of the study population. Following an optimal staging procedure $(n=41)$, a grade I tumour was diagnosed in 19 patients. According to the guidelines none of these

Table 2

Follow-up of the study population $(n=125)$

\begin{tabular}{|c|c|c|}
\hline & $\begin{array}{l}\text { Optimal } \\
\text { staged }\end{array}$ & $\begin{array}{l}\text { Non-optimal } \\
\text { staged }\end{array}$ \\
\hline $\begin{array}{l}\text { Total follow-up } \\
\text { (person-years) }\end{array}$ & 266.6 & 468.0 \\
\hline $\begin{array}{l}\text { Mean follow-up } \\
\quad \text { (years) }\end{array}$ & 6.5 & 5.6 \\
\hline $\begin{array}{l}\text { Median follow-up } \\
\quad \text { (years) }\end{array}$ & 6.0 & 5.4 \\
\hline Range & 2.7 years-11.1 years & 1 week-11.1 years \\
\hline $\begin{array}{l}\text { Total number of } \\
\text { deaths }(n(\%))\end{array}$ & $2(4.9)$ & $29(34.5)$ \\
\hline $\begin{array}{l}\text { Due to ovarian } \\
\text { cancer }(n(\%))\end{array}$ & $1(2.4)$ & $13(15.5)$ \\
\hline $\begin{array}{l}\text { Not due to } \\
\quad \text { ovarian ca. }(n(\%))\end{array}$ & $1(2.4)$ & $16(19.0)$ \\
\hline
\end{tabular}

Table 3

Steps performed in the surgical staging procedure in 41 optimally staged patients and 84 non-optimally staged patients

\begin{tabular}{|c|c|c|c|c|}
\hline & \multicolumn{2}{|c|}{$\begin{array}{l}\text { Optimally } \\
\text { staged }\end{array}$} & \multicolumn{2}{|c|}{$\begin{array}{l}\text { Non-optimally } \\
\text { staged }\end{array}$} \\
\hline & $n$ & $(\%)$ & $n$ & $(\%)$ \\
\hline $\begin{array}{l}\text { Bilateral salphingo- } \\
\text { oophorectomy }\end{array}$ & 32 & 78.0 & 70 & 83.3 \\
\hline $\begin{array}{l}\text { Transabdominal } \\
\text { hysterectomy }\end{array}$ & 32 & 78.0 & 69 & 82.1 \\
\hline Infracolic omentectomy & 41 & 100.0 & 55 & 65.5 \\
\hline $\begin{array}{l}\text { Random peritoneal and } \\
\text { diaphragm biopsies }\end{array}$ & 41 & 100.0 & 36 & 42.9 \\
\hline $\begin{array}{l}\text { Pelvic lymph node } \\
\text { sampling or dissection }\end{array}$ & 41 & 100.0 & 13 & 15.5 \\
\hline $\begin{array}{l}\text { Paraaortic lymph node } \\
\text { sampling or dissection }\end{array}$ & 41 & 100.0 & 19 & 22.6 \\
\hline Abdominal washings & 41 & 100.0 & 47 & 56.0 \\
\hline $\begin{array}{l}\text { One surgical intervention } \\
\text { (total 86) }\end{array}$ & 14 & 11.2 & 72 & 57.6 \\
\hline $\begin{array}{l}\text { Two surgical interventions } \\
\text { (total 39) }\end{array}$ & 27 & 21.6 & 12 & 9.6 \\
\hline
\end{tabular}

patients did receive adjuvant therapy. Twenty-two patients did have an indication for adjuvant therapy, i.e. a grade II/III or clear cell tumour. However, only 11 patients did receive radiotherapy and 4 did receive chemotherapy.

In the non-optimally staged group (84 patients) all 43 patients with a grade I tumour did not receive adjuvant therapy. Only 29 of the 37 patients with an indication for adjuvant therapy according to tumour histology actually did receive it. In four of the non-optimally staged patients the histological grade of the tumour was unknown. Therefore, it was not possible to evaluate whether the guideline was followed.

One hundred and twenty-five patients contributed to a total of 734.6 person years of follow up. In total 31 of the 125 patients died $(24.8 \%)$. Thirteen patients died due to ovarian carcinoma. For 18 patients the cause of death was not related to the ovarian carcinoma. One patient died 1 week after diagnosis of ovarian carcinoma, because of a pulmonary embolism. We were not able to trace five patients to evaluate if they were still alive on 1st January 2002.

Kaplan-Meier analysis yielded 5-year overall survival figures in the optimally and non-optimally staged groups of 97.6 and $68.5 \%$, respectively. The 5-year overall survival figures for women with a histological grade I disease were $100.0 \%$ in the optimally staged group $(n=19)$ and $81.4 \%$ in the non-optimally staged group $(n=43)$.

For women with a histological grade II or III disease these 5-year overall survival figures were $95.5 \%(n=22)$ and $49.5 \%(n=37)$, respectively.

The survival difference of $29.1 \%$ between the optimally and non-optimally staged groups is disturbed by the cofounders age and histological grade. To adjust for these factors we used a Cox Proportional Hazard model. Table 4 shows the results. 


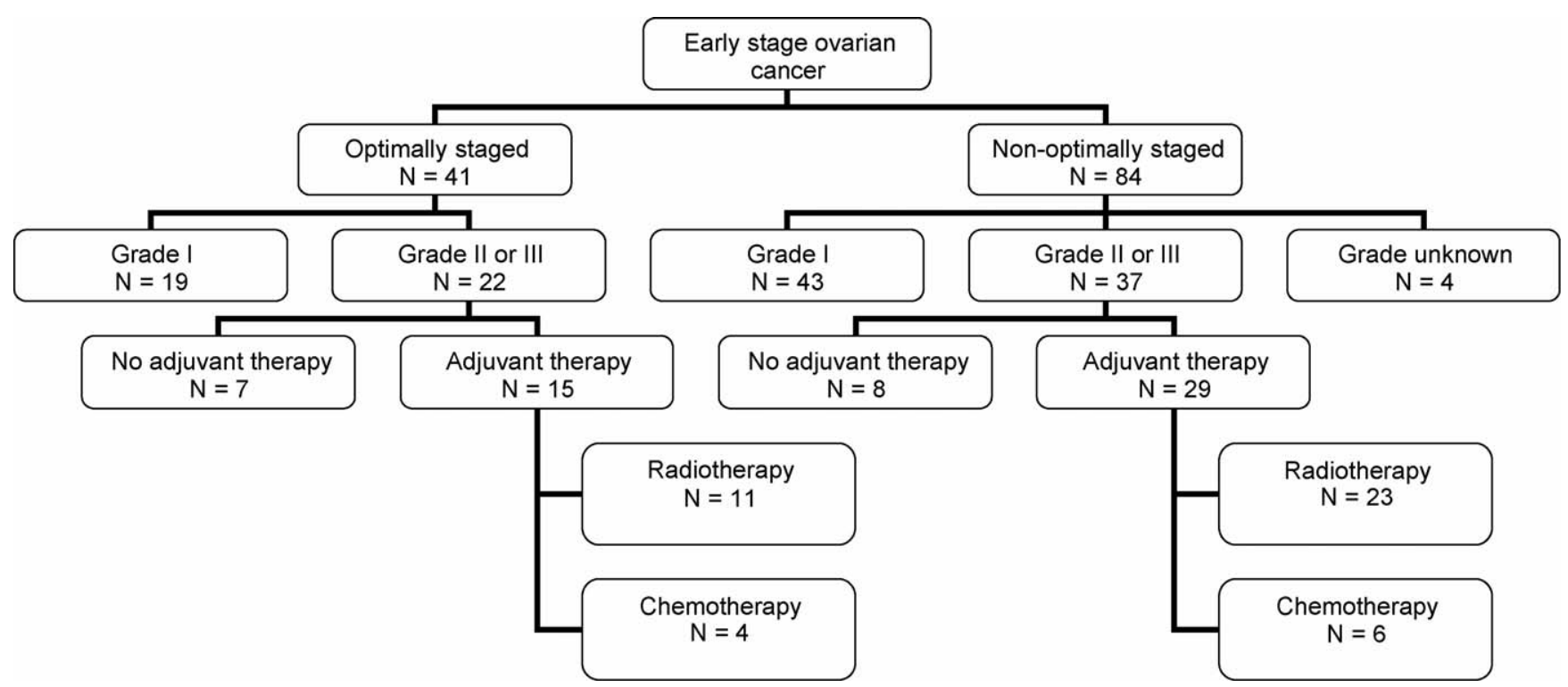

Fig. 1. Adjuvant treatment of 125 patients with early-stage ovarian cancer.

Table 4

Cox Proportional Hazard model for risk of death due to all causes $(n=121)$

\begin{tabular}{lll}
\hline & HR & CI (95\%) \\
\hline Surgical staging conform guidelines & 7.4 & $1.7-32.2$ \\
Age & 1.0 & $1.0-1.1$ \\
Histological grade: II and III comparing to I & 3.8 & $1.7-8.3$ \\
\hline
\end{tabular}

Included were: age, stage, grade, histological type, surgical staging.

Histological grade was reduced into two categories, which is grade I versus grade II/III and clear cell. Patients, who were sub-optimally staged, had a significant higher risk to die than patients who were optimally staged did (HR: 7.4; 95\% CI: 1.7-32.2).

Adjuvant therapy administered according to the guideline did not improve overall survival in patients with grade II/III or clear cell tumours. In the optimally staged group, two patients died. In one of them death was due to ovarian carcinoma. Both patients had adjuvant therapy as prescribed in the guidelines. In the non-optimally staged group, 29 patients died. Six of them did not get adjuvant therapy although it was indicated according to the guidelines, whereas the remaining 23 patients were treated as prescribed by the guideline.

\section{Discussion}

In this study, compliance to the guideline for staging surgery for early-stage epithelial ovarian carcinoma was poor. Only in $32.8 \%$ of patients the staging procedure was performed correctly. Reasons for not following the guideline could not be retrieved from the medical records in $82 \%$. In most cases, optimal staging required a second surgical procedure (Table 3 ). Old age might have limited the number of patients eligible for secondary surgery. There is, however, no statistical difference in mean age, nor in range between staged and unstaged patients (Tables 1 and 4).

Guidelines for adjuvant therapy were followed in all grade I patients and in $74.6 \%$ of the grade II and III patients. In clinical practice a dilemma could arise when the diagnosis of epithelial carcinoma is posed on an extirpated ovarian cyst. Either the clinician can offer the patient a second surgical intervention or adjuvant therapy. This dilemma is aggravated when the guideline for adjuvant therapy already requires adjuvant therapy on the basis of tumour differentiation. Theoretically, one can argue in such cases that the indication for a second intervention disposes.

To elucidate the value of adjuvant chemotherapy in early stage ovarian carcinoma patients the ICON I and ACTION trial were designed [12-14]. After initial surgery, adjuvant platinum-based chemotherapy was compared with observation. In the ACTION trial only one-third of the patients had adequate staging procedures in the observational arm as well as in the adjuvant therapy arm. This is comparable to our study. Analyses on overall survival and recurrence free survival were made of each trial separately as well as for the combined trials. In the ACTION trial no difference in survival was seen between the two trial arms. When optimally staged patients were analysed chemotherapy. In the analyses of the ICON I and in the combined trial a survival benefit is found for patients in the adjuvant therapy arm compared to patients in the observational arm. Unfortunately, separate analyses between the optimally staged and non-optimally staged groups could not be done.

Benefits from adjuvant therapy might therefore only reflect the amount of unappreciated residual disease in nonstaged patients. It may be carefully concluded that optimally 
staged patients do not benefit from adjuvant therapy, whereas non-optimally staged patients as a group do. Our guidelines prescribe observation only in case of a grade I tumour. In all other cases (grade II, III and clear cell tumours) adjuvant therapy is indicated. This may tempt the clinician to skip the second surgical procedure, necessary for optimal staging. At least, when adjuvant therapy is considered to be an adequate replacement for staging. For 49 patients in our study this could have been the reason to act in discordance to the guideline.

The actual question therefore is whether an adequate comprehensive optimal staging procedure after initial surgery can outweigh adjuvant chemotherapy.

This issue is studied by Le et al. [15]. Two treatment regimens are compared. After initial surgery one group of patients had an optimal staging procedure. Occult extraovarian disease was found in $36 \%$ of patients who underwent complete staging. The other group was not staged but either was managed expectantly or received chemotherapy on the basis of high risk factors (dense adhesions, high tumour grade, disease at the surface of the ovary or large areas of necrosis). Of the patients who were staged as a stage I after optimal staging, recurrent disease was seen in 10\%. Among those who were not staged and had no risk factors and did not receive adjuvant therapy, recurrence was seen in $28 \%$ $(p=0.036)$.

A survival benefit (68\% versus 58\%) was also found for the group of patients who were optimally staged versus the high-risk non-staged patients. The optimally staged group consisted of 'real' stage I patients as well as those who were upstaged (stages II and III) Another remarkable finding is that there was a trend toward worsened survival in the staged group receiving chemotherapy (truly stages II and III patients) compared to the high-risk un-staged group receiving chemotherapy. The authors explain this phenomenon that in the un-staged group true stage I patients are over treated. If this is true adjuvant chemotherapy cannot replace optimal staging!

Unfortunately, the numbers in this study are too small to draw hard conclusions; nevertheless we have to keep this warning in mind when we treat our patients.

In our study compliance to the guideline for surgery has, in accordance with the above-described study, a significant influence on overall survival of early epithelial ovarian cancer. Kaplan-Meier analysis yielded a difference of $29.1 \%$ (95\% CI: 26.1-32.1\%) in 5-year survival figures between the complete and incomplete staged groups. I.e., the un-staged group contain many patients with occult disease who are not treated adequately.

All studies, including our own, show consistently the value of optimal staging in early stage epithelial ovarian carcinoma. Therefore, more efforts should be put into the implementation of existing guidelines. A change in doctor characteristics combined with easy access to a gynaecological-oncologist, supported by the CCC, may help to raise the number of optimally staged women.
Like the ACTION trial but contrary to the ICON 1 and the combined studies, our study did not show a benefit of adjuvant therapy. In our study, similar to the ACTION trial, patients with a well-differentiated stages Ia and Ib tumour were excluded from adjuvant therapy, while in the ICON 1 study it is not clear whether these patients participated (in our study patients with Ic well differentiated tumours were also excluded). One reason can be that in our study two/third (22 versus 37 ) of the patients with an indication for adjuvant therapy were optimally staged, in contrast to the other studies. Another possibility is the number of patients who received radiotherapy instead of platinum based chemotherapy. Most centres switched to chemotherapy after platinum was introduced. A few papers [16,17] describe similar results for whole abdominal radiotherapy as chemotherapy. However, the number of patients in our study who had an indication for adjuvant therapy may have been too small to show such a difference. Moreover, data from our study were not derived from a randomised controlled clinical trial. Nevertheless, we feel that our study makes a valid contribution since it reflects current treatment practise in a well-defined area where all potential study subjects were included and it evaluates the compliance to guidelines in regard to patient survival in early-stage ovarian cancer.

Apart from all this the results also support the Dutch gynaecological oncology guidelines regarding treatment of early ovarian cancer [18].

In regard to the guideline for adjuvant therapy additional trials are needed to better identify patients who will benefit from this therapy. Recent studies, including our own, show results that emphasize the need for optimal staging in order to limit the use of chemotherapy in an adjuvant setting.

\section{Conclusions}

Compliance to the guideline for surgery is poor in patients with clinically early-stage ovarian carcinoma in the CCCMN region in The Netherlands. The guideline for adjuvant therapy also is insufficiently followed. Reasons why treatment deviated from the guidelines could often not be retrieved from the medical records. Comparisons are made with other populations described in literature. Possible reasons for the distressing compliance to the guidelines are discussed. A considerable advantage in survival was found in the group of patient treated as indicated by the surgical guidelines, while no benefit occurred in patients treated with adjuvant chemotherapy.

As a result we would like to stress that optimal surgical staging in early-stage epithelial ovarian cancer is paramount. Further studies are needed to define high-risk factors to indicate adjuvant chemotherapy. More research is urgently needed to identify factors that prevent compliance to the 
guideline for early-stage ovarian cancer in daily practise, and to eliminate these factors.

\section{Acknowledgements}

The authors would like to acknowledge Diakonessenhuis (3508 TG Utrecht), Hofpoort Hospital (3440 JD Woerden), Meander Medical Center (3800 BM Amersfoort), Mesos Medical Center (3503 RP Utrecht), Rivierenland Hospital (4000HA Tiel), Sint Antonius Hospital (3430 EM Nieuwegein), UMCU University Medical Center Utrecht (3508 GA Utrecht) for their support.

\section{References}

[1] Berg WN vd, Eliel MR, Batterman JJ (editors). Oncologieboek, tumorspecifieke richtlijnen, deel 1. Van Wijland, Laren; 2002. ISBN: 90-5453-006-5.

[2] Faught W, Lotocki RJ, Heywood M, Krepart GV. Early ovarian cancer: value of a negative staging laparotomy. Eur J Gynaecol Oncol 1996; 17:200-3.

[3] Hoskins WJ. Surgical staging and cytoreductive surgery of epithelial ovarian cancer. Cancer 1993;71(Suppl):1534-40.

[4] WHO: International classification of diseases for oncology. 1st ed. La Concorde, Geneva; 1976, ISBN: 92-4-154056-7.

[5] Le T, Adolph A, Krepart GV, Lotocki R, Heywood MS. The benefits of comprehensive surgical staging in the management of early-stage epithelial ovarian carcinoma. Gynecol Onc 2002;85:351-5.

[6] Schilder JM, Thompson AM, DePriest PD, et al. Outcome of reproductive age women with stage $1 \mathrm{~A}$ or $1 \mathrm{C}$ invasive epithelial ovarian cancer treated with fertility-sparing therapy. Gynecol Oncol 2002; 87:351-5.
[7] Di Re F, Fontanelli R, Raspagliesi F, Di Re E. Pelvic and para-aortic lymphadenectomy in cancer of the ovary. Bailleres Clin Obstet Gynaecol 1989;3:131-42.

[8] Benedetti-Panici P, Greggi S, Mneschi F, et al. Anatomical and pathological study of retroperitoneal nodes in epithelial ovarian cancer. Gynecol Oncol 1993;51:150-4.

[9] Dembo AJ. Radiation therapy in the management of ovarian cancer. Clin Obstet Gynecol 1983;10(2):261-78.

[10] Einhorn N, Bjokholm E, Krebs I, Petterson F, Tjernberg B. Treatment in early stages of ovarian carcinoma. A randomized study. Nordisk for Obstetrik och Gynekol 1980;2:65.

[11] Thomas GM. Radiotherapy in early ovarian cancer. Gynecol Oncol 1994;(55(3Pt2)):S73-9.

[12] Trimbos JB, Vergote I, Bolis G, et al. Impact of adjuvant chemotherapy and surgical staging in early-stage ovarian carcinoma: European organisation for research and treatment of cancer: Adjuvant Chemotherapy in Ovarian Neoplasm trial. Natl Cancer Inst 2003;95:113-25.

[13] Trimbos JB, Parmar M, Vergote I, et al. International collaborative ovarian neoplasm trial 1 and adjuvant chemotherapy in ovarian neoplasm trial: two parallel randomized phase III trials of adjuvant chemotherapy in patients with early-stage ovarian carcinoma. J Natl Cancer Inst 2003;95:105-12.

[14] International Collaborative Ovarian Neoplasm 1 (ICON 1). International collaborative ovarian neoplasm trial 1: a randomized trial of adjuvant chemotherapy in women with early-stage ovarian cancer. J Natl Cancer Inst 2003;95:125-31.

[15] Le T, Krepart GV, Lotocki RJ, Heywood MS. Clinically apparent early stage invasive epithelial ovarian carcinoma: should all be treated similarly? Gynecol Oncol 1999;74:252-4.

[16] Morton GC, Thomas GM. Is there a place for Whole Abdominal Radiotherapy in the management of ovarian cancer? Ann Acad Med Singapore 1996;25(3):429-36.

[17] Skirnisdottir I, Sorbe B. Adjuvant radiotherapy in stages I-II epithelial ovarian cancer. Eur J Gynaecol Oncol 2001;22(6).

[18] http://www.oncoline.nl/. 